\title{
XXXV. On the electrification of gases exposed to Röntgen rays, and the absorption of Röntgen radiation by gases and vapours
}

\section{E. Rutherford M.A.}

To cite this article: E. Rutherford M.A. (1897) XXXV. On the electrification of gases exposed to Röntgen rays, and the absorption of Röntgen radiation by gases and vapours, Philosophical Magazine Series 5, 43:263, 241-255, DOI: 10.1080/14786449708620988

To link to this article: http://dx.doi.org/10.1080/14786449708620988

曲 Published online: 08 May 2009.

Submit your article to this journal $₫$

Џ Article views: 12

Q View related articles ¿ 
LONDON, EDINBURGH, AND DUBLIN

\title{
PHILOSOPHICAL MAGAZINE
}

\author{
AND \\ JOURNAL OF SCIENCE.
}

[FIFTH SERIES.]

APRIL 1897.

XXXV. On the Electrification of Gases exposed to Röntgen Rays, and the Alsorption of Röntgen Radiation by Gases and Vapours. By E. Rutrerford, M.A., 1851 Exhibition Science Scholar, University of New Zealand, Trinity College, Cambridge*.

TN a recent paper by Prof. J. J. Thomson and myself " On 1 the Passage of Electricity through Gases exposed to Röntgen Rays" (Phil. Mag. Nov. 1896), a method of obtaining electrified air by means of the Röntgen rays was very briefly explained.

The present paper deals with further experiments which have been made to investigate more fully the way in which electrified gases can be obtained by means of the Röntgen rays, and also to examine the properties of the charged gas. The opacity of gases for Röntgen radiation has also been examined.

A gas becomes a temporary conductor under the influence of the Röntgen rays, and preserves its power of conducting some short time after the rays have ceased to act; since the conduction in the gas is probably due to the convection of charged particles which travel through the gas with a velocity of the order of $1 \mathrm{~cm}$. a second for a potential gradient of one volt per cm., it is not surprising that we can separate the positive from the negative conducting particles before they give up their charges to the electrodes.

* Communicated by Prof. J. J. Thomson, F.R.S.

Phil. Mag. S. 5. Vol. 43. No. 263. April 1897. U 
The method of separation used was to direct a rapid current of air or other gas along the surface of the charged electrode of a vessel exposed to the Röntgen rays; a large metal cylinder was taken, either of thin metal to allow the rays to readily pass through the side, or a piece was cut out and a sheet of very thin metal substituted to serve the same purpose.

Fig. 1.

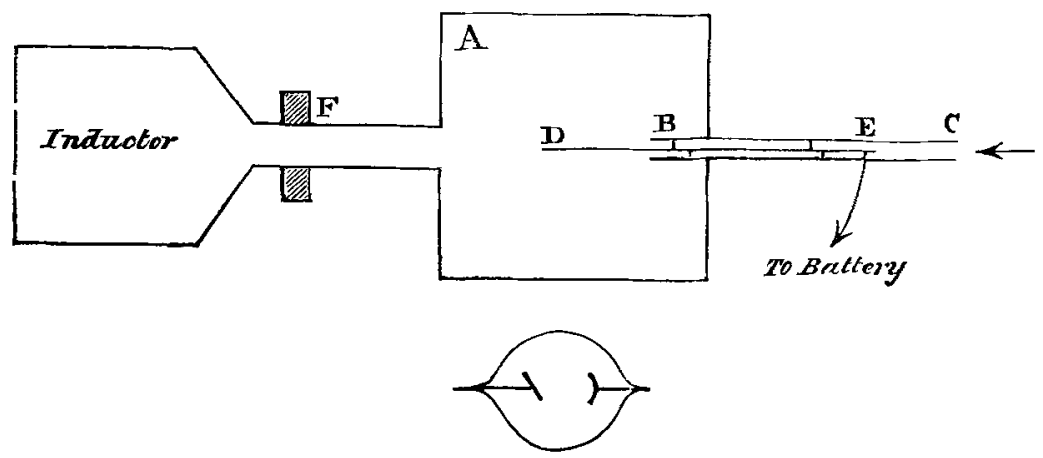

A (fig. 1) was the metal cylinder, $\mathrm{B} \mathrm{C}$ a glass tube fixed centrally inside the cylinder. The wire $\mathrm{DE}$ was fixed in the glass tube $\mathrm{BC}$ and supported by thin metal spikes in the centre of the tube. Several inches of wire, BD, projected from the glass tube. A current of gas was sent from a pair of bellows or a gas reservoir along the tube CB, and then along a metal tube into an insulated conductor connected with one pair of quadrants of an electrometer, the other pair being connected to earth. The wire $\mathrm{DE}$ was connected to one pole of a battery of small lead cells, the other pole being to earth. The outside of the cylinder was connected to earth, and the bulb and Ruhmkorff coil were placed inside a metal tank, so as to completely screen the outside apparatus from electrostatic disturbances. A hole was cut in the tank, and the bulb arranged so as to allow the rays to fall on the part $\mathrm{BD}$ of the charged wire.

When the bulb was not working, however rapid a current of air was sent along the charged electrode, no electrification was obtained in the inductor, but the moment the rays were turned on the inductor became charged opposite in sign to the charged wire. The deflexion of the electrometer continuously inceased as long as the rays and blast of air were acting.

The inductor was generally placed some feet from the generating vessel $A$, the air passing to the inductor through a metal tube of $3 \mathrm{~cm}$, diameter. Since the electrification of 
the inductor is opposite in sign to the charged wire, the effect can in no way be due to conduction through the Röntgenized air from the charged electrode to the inductor, since the inductor would then be charged to the same sign as the electrode.

A small plug of glass wool placed in the metal tube between the generator and the inductor completely stopped all electrification, and the inductor received no charge, however rapid a blast of air was sent through the apparatus.

On account of the large quantity of air blown through the inductor in order to obtain a convenient deflexion on the electrometer (an amount sufficient to fill the inductor many times over), only a small proportion of the charge could be blown out. If, however, a gentle current of electrified air was blown into the inductor for two or three seconds, and the rays then stopped, it was found possible to blow out most of the charge again, after a short interval, provided there was a fairly wide opening in the inductor. If the opening was stopped with a plug of glass wool, it was found impossible to blow out the slightest amount, since the electrified particles gave up their charge freely to the glass wool.

Since the glass wool has the power of completely discharging the electrification both positive and negative, a short wide metal cylinder lightly packed with glass wool was used instead of the inductor for testing the amount of the electrification in most of the experiments that follow.

The amount of electrification obtained varied with the potential of the charged wire and the velocity of the current of air. The relation between the amount of electrification and the E.M.F. of the charged wire is shown in the Table below :-

\begin{tabular}{|c|c|}
\hline E.M.F. in volts. & $\begin{array}{c}\text { Amount of Electrification } \\
\text { in scale-divisions. }\end{array}$ \\
\hline 17 & 60 \\
35 & 100 \\
70 & 130 \\
200 & 82 \\
\hline
\end{tabular}

The amount of electrification increases up to a certain point and then diminishes. The maximum amount of electrification is closely connected with the value of the E.M.F. which is just sufficient to give the saturation-value of the 
current through the gas. In the above table the saturationvalue of the E.M.F. was about 70 volts, and this corresponds to the maximum amount of electrification.

Since the velocity of the conducting particles increases with the E.M.F. but the current through the gas remains constant, when the E.M.F. is raised above its saturationvalue it is to be expected that a greater proportion of the conducting particles would reach the electrode. This agrees with experiment, for as the E.M.F. is increased above a certain value the amount of electrification obtained steadily diminishes.

The amount of electrification obtained for a given E.M.F. increases at first with the velocity of the blast, and then tends to a maximum value, which cannot be increased, however rapid a blast is sent along the wire.

An experiment proving conclusively that the amount of electrification is intimately connected with the conduction of electricity through the gas is as follows :-The electrode along which the air was blown was carefully insulated and connected to one pair of quadrants of the electrometer. The two pairs of quadrants were charged up to the same potential and then insulated from each other, and the rate of leak of the charged wire determined. The rate of leak steadily diminished with increase of velocity of the blast; when the air issuing from the glass tube bad a velocity of about $1000 \mathrm{~cm}$. per second the rate of leak was only one fourth of its value when the air was still, and the amount of electrification in the air passing from the wire, as tested by the glass wool cylinder, was nearly equal to the quantity of electricity corresponding to the difference between the two rates of leak.

We should not expect them to be exactly equal, since some of the Röntgenized air containing both positively and negatively charged particles is also blown out.

The charged gas obtained in this way is thus due to an excess of the positive or negative conducting particles, whatever they may be, to which conduction in gas under the Röntgen rays is due.

In all these exr priments precautions were taken against dust. It was found that the amount of electrification obtained was independent of the quantity of dust in the air provided the velocity of the issuing blast was kept constant. The air in one case was sent through a long tube filled with glass wool into the gas reservoir, which was then allowed to stand for a couple of days without being disturbed. The air in passing from the reservoir to the generator was again passed through glass wool, but the effect obtained was exactly the same as if 
the dust-charged air from the room were sent directly through the apparatus.

\section{Electrification from Charged Insulators.}

If the central electrode through which the air was blown was coated with paraffin or sealing-wax, it was found that the amount of electrification obtained was at first about equal to the amount with the bare electrode. If the bulb was kept working the amount of electrification diminished after a time. The central electrode was then connected to earth, and when the $x$-rays were acting electrification could still be obtained, but of opposite sign to that obtained before. If the wire with the coating of dielectric on its surface was kept charged to a high potential and the rays continued for some time, on applying a smaller E.M.F. to the wire in the same direction the sign of the electrification is generally changed.

The explanation of these and similar phenomena is simple if we consider that the conducting particles of the gas either give up their charge to the surface of the insulator, or adhere to the surface which becomes charged opposite in sign to the wire itself. If the bulb is kept working, the electromotive intensity acting on the gas is diminished, owing to the effect of the oppositely charged insulator. The amount of electrification obtained theretore diminishes if the E.M.F. is not well above the saturation-value. If the central electrode be then connected to earth, the charged insulator causes a current through the gas in the opposite direction, and thus changes the sign of the charge in the gas blown out. If the charge on the insulator is large, as is the case if the central wire has been raised, for example, to a potential of 200 volts and exposed to the rays for some time, on applying an E.M.F. of 30 volts, say, in the same direction the electrification changes sign. In this case, the electromotive intensity due to the charged insulator is greater and opposite in sign to that due to the 30 volts, and so the current through the gas is reversed.

The sign of the electrification obtained when the wire is covered with insulating material is thus dependent on the amount and sign of the charge on the surface of the dielectric.

From a charged wire coated with paraffin or sealing- wax which had been exposed to the Röntgen rays for several minutes, it was found possible to obtain electrified air, by directing a current of air along its surface, several hours after the central electrode had been connected to earth. 


\section{Properties of the Charged Gas.}

Since the charged gas obtuined is due to the separation of the oppositely charged conducting particles to which conduction in a gas under the $x$-rays is probably due, we should expect the positively and negatively electrified gas to closely resemble Röntgenized air in its properties, and such is found to be the case.

The gas completely loses its charge in its passage through the pores in a plug of glass wool; while Röntgenized air, after being forced through glass wool, loses all trace of conductivity.

The gas readily gives up its charge to any conducting or insulating surface against which it impinges. The greater amount of electrification is discharged in the passage of the gas down a long tube. If the electrified air is allowed to impinge against the surface of an insulated metal plate, it gives up a portion of its charge to the metal. The facility with which the gas is discharged is to be expected, since no evidence of polarization has been found in the conduction of the gas exposed to the Röntgen rays when metal electrodes are used.

A remarkable property of the electrified gas is that positive and negative electrification are not discharged with equal facility by all metals. When the charged gas was passed through a long zinc tube, the amount of negative electrification on the issuing gas was always less than the amount of positive for the same velocity of the blast. By insulating the vinc tube it was found that it received a greater charge of negative than of positive. In order to test this difference, cylinders of zinc, tin, and copper were made of the same size, and the charged gas forced through them. It was found that zinc and tin discharged negative electrification more rapidly than positive, the difference in general amounting to about 20 per cent. Copper apparently discharged the positive and negative with about equal facility, but many experiments seemed to point to the conclusion that even in the case of copper negative was slightly more readily discharged than positive. If the electrified gas impinged against insulated plates of different metals, the same general results were obtained.

Not only was there a difference in the discharging powers of positive and negative electrification for any particular metal, but a copper plate, for example, discharged positive more readily than a zinc plate placed exactly in the same 
position, while the zinc plate discharged more negative than the copper.

Other metals, like aluminium, lead, were tested, and in all cases negative electricity was discharged with slightly more facility than positive.

The variable discharging power of the different metals agrees in some respects with the results obtained by Minchin ('Electrician,' March 27, 1896), who found that under the influence of the Röntgen rays insulated metal plates were all charged up to a small potential. According to his results copper was charged positively and zinc negatively, while sodium was highly negative. He also found that the potential to which some of the metals could be raised depended on the degree of polish of the exposed surface. In the experiments on the discharging power of the metals, the results were dependent to some extent on the brightness of the surface, especially in the case of tin and zine.

'The amount of electrification discharyed by a metal tube one inch in diameter and a foot long is very large, amounting in some cases to over one fourth of the whole charge on the gas. It must be remembered, however, that the current of air conveying the charged gas is travelling at a high velocity, and is, in consequence, in a state of violent eddying motion, so that probably a large proportion of the gas approaches near the surface in its passage along the tube.

The charge is taken from the gas not only when it passes through metal tubes, but also when it passes through tubes coated with an insulator. A metal tube was taken and coated with a thin layer of paraffin, and it was found that the charge on the cylinder was about the same as with the clean metal. It was difficult to determine with certainty whether insulators exhibited similar properties to metals in regard to discharging power. The amounts of positive and of negative electrification dissharged were approximately the same, but the differences were too small to make certain of.

The conductivity of the charged gas was tested by placing an insulated wire kept at a constant potential inside a metal vessel through which the electrified gas was blown. It was found that when the electrification was of the same sign as the charged wire, the gas gave up its charge to the outside vessel, and when of the opposite sign, to the charged wire. The current tbrough the gas was only temporary, and ceased as soon as the current of electrified gas was stopped. 


\section{Electrification of Different Gases.}

All the gases which were experimented with could be electrified in the same way as air. A gas-bag was filled with the gas to be tested, and then forced along the electrode as in the case of air, care being taken to allow the gas to run through some time before the rays were turned on, in order to remove the air as far as possible from the generating vessel.

The amounts of electrification obtained for a given velocity of the gas and intensity of the rays varied with the conductivity of the gas under the $x$-rays. Gases that have a greater conductivity than air gave more electrification than air. Oxygen and coal-gas gave slightly less electrification than air, while carbon dioxide gave slightly more-the amounts being sensibly proportional to their conductivities.

The vapour of methyl iodide was tried, which bas a very high conductivity-over 20 times that due to air. Only a partial test could be made of it, as sufficient quantity of the vapour was not obtainable. Some of the liquid was placed in the generator (fig. 1), and gently heated to its boilingpoint, till the vessel was filled with vapour. The rays were then turned on, and a rapid current of air sent for 3 or 4 seconds along the central electrode. The amount of electrification obtained was over 5 times the amount from air in the same time. After a few seconds the highly conducting vapour was blown out and the electrification became sensibly that due to air alone. If a current of the vapour could have been sent instead of a current of air, it is probable that the amount of electrification obtained would have been over 20 times that of air in the same time.

The experiments on hydrogen were interesting as bearing on the question of the relative velocity of the conducting particles of hydrogen and air. For a given small weight on the gas-bag the amount of electrification from air was 2.5 times that due to hydrogen in the same time. As the weight was increased the ratio fell to $1 \cdot 5$. This is as we should expect if the velocity of the hydrogen ions was greater than those of air. For small velocities of the blast a nuch smaller proportion of the hydrogen than the air ions escape. As the velocity is increased the amount of electrification from the air increases slowly, as nearly all of the ions are blown out, while the amount from hydrogen increases rapidly as the velocity increases.

In a previous paper (loc. cit.) it was shown that hydrogen was saturated for a much lower value of the E.M.F. than 
air, while the velocity of the hydrogen ion was much greater than that of air. The experiments on the amount of electrification with variation of E.M.F. and velocity of the blast contirm the previous results which were obtained in an entirely different way.

Velocity of the Ions. - An approximate determination of the velocity of the conducting particles for air can be made by determining the rate of leak of a charged wire connected to an electrometer when air is blown at varying velocities from a tube of known diameter along the charged wire. If we assume the current of air of high velocity from the tube to be confined within narrow limits for a short distance from the orifice, the velocity of the ions in order that a known proportion of the ions should reach the electrode can easily be deduced. The velocity of the blast issuing from a tube $8 \mathrm{~cm}$. in diameter was $800 \mathrm{~cm}$. per second, and the wire was charged to a potential of 35 volts. With this velocity the rate of leak of the wire was only one third of the natural leak; so that two thirds of the conducting particles of one sign were blown out. The length of the exposed wire B D (fig. 1) was $6.3 \mathrm{~cm}$., and knowing the diameter of the wire $B D$ and of the cylinder, it can be shown that the velocity of the conducting particles for air is about $1 \mathrm{~cm}$. per second for a potential gradient of one volt. This is of the same order as the rough determination made in the previous paper by Professor Thomson and myself.

The positive and negative conducting particles of air travel with the same velocity, for when the sign of the charged wire is reversed the rate of leak is the same as before with the same velocity of the blast. When different amounts of positive and negative electrification were obtained, it was at first thought that part of the difference might be due to inequality in the velocity of the ions, but later experiments showed that it was entirely due to the greater facility with which metals discharged the negative electrification.

\section{Volume-Density of Electrification of the Charged Gas.}

Only a very minute portion of the gas conveys the charge in the cases we have been considering. In the paper previously referred to it has been shown that assuming the conducting particles convey an atomic charge, only about one billionth of the gas is required to be split up to give the conductivity observed. In the previous experiments the conducting air is still further diluted by the blast along the electrode which conveys the charged particles with it. From data of the capacity of the electrometer and velocity of the blast it can be shown that the amount of charge per c.c. 
of air was about $10^{-4}$ electrostatic units. In the case of the better conducting gases and vapours the volume-density is greater. For the vapour of methyl iodide the volumedensity would be over 20 times as great.

The facility with which the electrified gas is discharged by metals and insulators may at first sigbt lead to the conclusion that we are dealing with electrified dust, which, as is well known, is completely discharged by glass wool, and also readily gives up its charge to whatever it comes in contact with. It has been shown, however, that the amount of electrification is quite independent of the amount of dust in the air, and that therefore the electrification can be in no way due to electrified dust.

The theory has been advanced that the discharge of electricity from the surface of a metal under the action of ultraviolet light is due to the disintegration of metallic particles or vapour from the surface, and that these carry off the charge. The discharge of electrification by the Röntgen rays might possibly be due to a similar cause, and this was fully investigated. In the first place, there are many experiments which negative this view. It has been shown in a previous paper that the current through a gas conducting under the $x$-rays increases with the distance between the electrodes although the surface exposed to the gas is unaltered. The amount of electrification obtained from a gas was found to be quite independent of the nature of the electrodes. 'The inside electrode (fig. 1) of the cylinder was coated with paraffin or wax, and provided we do not allow the charge to collect on the surface of the insulator, the amonnt of electrification was unaltered. Similarly, if the inside of the cylinder was coated with an insulator, no difference in amount could be detected immediately after the rays were turned on; but after the rays had been acting for a short time the amount decreased, owing to the charging of the surfaces of the insulators. These conclusions show that the conductivity in a gas is independent of the nature of the surface of the electrodes, for it is extremely improbable that the same amount of dust would be dislodged by the rays from the surface of all metals and insulators.

The most conclusive experiments on this subject are some which I recently made on the diminution of the intensity of the Röntgen rays due to the absorption in their passage through gases and vapours.

\section{Alsorption of Energy by Gases and Vapours.}

Since gases all conduct under the influence of the $x$-rays, it was interesting to investigate the relative absorption in 
order to make the gases conductors, and whether the absorption was in any way related to the constitution or conductivity of the gas.

The absorption of energy in gases like air, hydrogen, oxy gen is small and is not easily detected unless a delicate null method is used.

Two equal and similar conical-shaped vessels $\mathrm{A} B \mathrm{C}, \mathrm{A}^{\prime} \mathrm{B}^{\prime}\left(\mathrm{C}^{\prime}\right.$ (fig, 2), much larger in diameter at the top than the bottom,

Fig. 2.
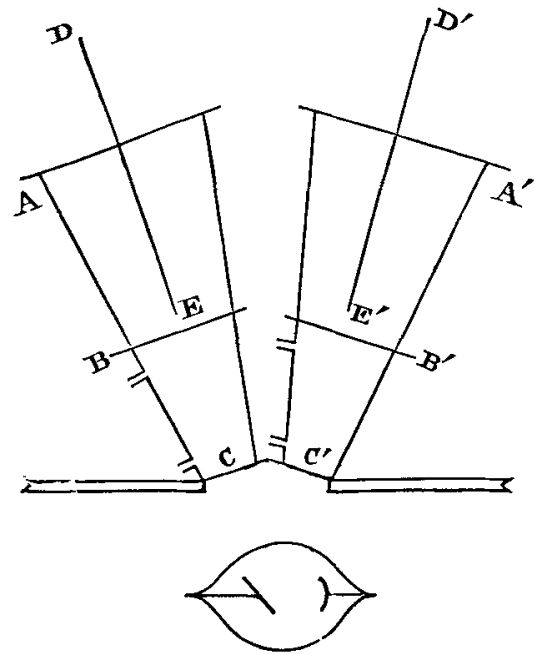

were placed in such positions that the axis of each cone passed as nearly as possible through the anode of the focusbulb. From experiments it was found that the $x$-rays appeared to emanate in all directions from the anode. The upper parts of each vessel, $A B, A^{\prime} B^{\prime}$, were made of lead, and were separated from the lower portions $\mathrm{BC}, \mathrm{B}^{\prime} \mathrm{C}^{\prime}$, which were made of glass, by thin ebonite plates. Thin ebonite plates also covered the ends of the glass cylinders at $\mathrm{C}$ and $\mathrm{C}^{\prime}$, so that the ressels $\mathrm{BC}, \mathrm{B}^{\prime} \mathrm{C}^{\prime}$ were air-tight, and could be exhausted when required.

The lead cylinders $\mathrm{A} \mathrm{B}, \mathrm{A}^{\prime} \mathrm{B}^{\prime}$ were used to compare the rate of leak after the rays had passed through the glass cylinders. Insulated wires $D \mathrm{E}, \mathrm{D}^{\prime} \mathrm{E}^{\prime}$ formed the electrodes, and these were connected to opposite pairs of quadrants of the electrometer, and both quadrants were at first charged up to the same potential, which in practice was generally 200 volts. The outsides of the vessels $A B, A^{\prime} B^{\prime}$ were connected to earth. 
The position of the bulb was so adjusted that the rate of leak in each cylinder was exactly the same, so that since the potential of each pair of quadrants fell at the same rate, the needle of the electrometer remained at rest while the rays were kept acting. If another gas was introduced into one of the glass vessels $\mathrm{BC}$ it was found that the balance was disturbed, owing to the variation of intensity of the rays in the vessel $A B$, which was caused by the less or greater absorption of the rays in their passage through the gas. In the experiments the only rays which caused conduction in the lead cylinders had to pass through the gas, and all stray radiation was carefully screened off.

If we assume that the absorption of energy in passing through a thin layer of gas of thickness $d l$ is proportional to the intensity of the rays I at that point and to the length of the gas $d l$ traversed, the decrease of intensity of the rays due to absorption of energy in the gas is equal to $\lambda \mathrm{I} d l$, where $\lambda$ is a constant for any particular gas but varies for different gases, and may be called the coefficient of absorption of the gas.

Experimentally it was found that the rate of leak of a gas is proportional to the intensity of radiation at any point.

From these considerations it can readily be shown that the ratio of the rate of leak when the rays pass through a length $l$ of the gas, to the rate of leak when the gas is removed and a vacuum substituted is $e^{-\lambda l}$ where $e=2 \cdot 7$, and this result is independent of any metal or insulators which the rays pass through in both cases before reaching the testing vessels.

The ratio of the rates of leak can be readily deduced from the morement of the electrometer needle, and since the length of the gas traversed is known, the coefficient $\lambda$ is thus determined.

Experiments were first made to see whether air absorbed any appreciable amount of energy of the radiation. The balance was obtained and then one of the glass vessels was exhausted by an air-pump; the electrometer slowly moved in one direction while the rays were kept acting. If the other vesäel was also exhausted, the balance was again restored, and if air was then let into the vessel first exhausted, the electrometer needle moved in the opposite direction. The variation of the rate of leak after passing through $10 \mathrm{~cm}$. of air was about one per cent., but it was a difficult matter to determine such a small variation with accuracy. . It will be at once seen that the approximate value of $\lambda l$ is $10^{-2}$, and therefore for air $\lambda=10^{-3}$, since $l=10 \mathrm{~cm}$.

If we suppose $x$-radiation to be emitted by the sun, assuming the radiation would have to pass through four miles 
of homogeneous atmosphere, the intensity at the surface of the earth would be approximately $10^{-260}$ of the intensity of the radiation before it reached the earth's atmosphere. This is an excessively minute proportion, and it is not surprising therefore that experiments, made even on the highest mountains, to detect any Röntgen rays in solar radiation (Cajori, Phil. Mag. Nov. 1896) should have been unsuccessful, even if the intensity of the $x$-radiation at the limits of our atmosphere were greater than could be produced at the surface of a Crookes tube.

Gases like oxygen, coal-gas, carbon dioxide, whose leakagerates are about the same as that of air, absorb about the same amount of energy.

Sulphuretted hydrogen, which has a conductivity six times as great as air, diminishes the intensity of the radiation by about 4 per cent. in passing through $10 \mathrm{~cm}$. of the gas. Chlorine, whose conductivity is eighteen times that of air, diminishes the intensity about 12 per cent. for the same distance.

The absorption of energy in these cases is not necessarily selective, for the same results were obtained whatever gas was used in the testing vessels. After the radiation had passed through sulphuretted hydrogen the same diminution in intensity was obtained whether air or sulphuretted hydrogen was used in the testing vessels.

Mercury vapour, which is one of the best conductors of electricity under the $x$-rays, is also one of the best absorbers of the radiation.

In the case of mercury it was not necessary to use a null method. A glass bulb $7 \mathrm{~cm}$. in diameter was taken and a small amount of mercury introduced. The bulb was slowly heated till it was filled with boiling mercury vapour, care being taken that no mercury was allowed to condense on the sides of the glass through which the rays passed. The rate of leak was then taken in a conlucting vessel for the radiation which had passed through the mercury vapour. The mercury was then removed and the bulb now filled with air was heated to the same temperature. The rate of leak in the testing vessel with air in the bulb was found to be twice as great as when the bulb wasfilled with mercury vapour at the temperature of boiling mercury.

In the passage therefore through $7 \mathrm{~cm}$. of mercury vapour sufficient energy is absorbed to reduce the intensity of radiation by one half.

The vapour of methy iodide, which is even a better conductor than mercury vapour, is also a powerful absorber of the radiation. 
When the temperature of the vapour was raised above the boiling-point of the liquid, the intensity of the radiation after passing through $13 \mathrm{~cm}$. of the vapour was only 4 of the intensity when the vapour was removed.

From experiments on the absorption of energy of different lengths of the vapour of methyl iodide, and also of the gases sulphuretted hydrogen and chlorine, the ratio of the intensity of radiation after passage through the vapour or gas to the intensity of the radiation when the gas was removed was found to be in agreement with the theoretical ratio $e^{-\lambda l}$. For short lengths of the gas the absorption is proportional to the length.

It has been shown that for the vapour of methyl iodide $e^{-\lambda l}=\cdot 4$ when $l=13 \mathrm{~cm}$; therefore $\lambda=\cdot 07$. The intensity of the radiation after passing through a length of 1 metre of the vapour is only one thousindth part of its value when the vapour is removed.

The absorption of energy varies with the pressure of the gas. The vapour of methyl iodide was used, and it was found that the values of $\lambda$ were roughly proportional to the pressures down to a pressure of one quarter of an atmosphere. Results of this kind, however, are difficult to determine with accuracy on account of the variation of the Crookes tube during a series of observations.

The following table gives the values of $\lambda$ and relative conductivities of some of the gases :-

\begin{tabular}{|c|c|c|}
\hline Gas. & $\lambda$. & Conductivitics. \\
\hline 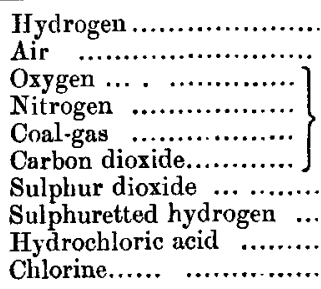 & $\begin{array}{r}\quad \begin{array}{c}(s m a l l) \\
.001\end{array} \\
\text { about } \cdot 001 \\
\\
.0025 \\
.0037 \\
.0065 \\
.0095\end{array}$ & $\begin{array}{l}\cdot 5 \\
1 \cdot 2 \\
\cdot 9 \\
\cdot 8 \\
1 \cdot 2 \\
4 \\
6 \\
11 \\
18\end{array}$ \\
\hline
\end{tabular}

These experiments show that good conductors under the $x$-rays are good absorbers of the radiation. The absorbing powers for the gases examined bad the same relative order as their conductivities. 'T he absorption does not seem to depend to any great extent on the molecular weight of the gas. Hydrochloric acid is nearly twice as good an absorber as sulphuretted bydrogen, although their densities are nearly equal; while it is more than ten times as good an absorber as 
carbon dioxide, a gas of greater density. It is interesting to observe that vapours like mercury and methyl iodide which allow light to piss through freely are very opaque to Röntgen radiation.

Since the absorption of energy of the radiation varies with the length of the gas traversed and with the conductivity of the gas, it is rery strong evidence that the discharge of electrification by the Röntgen rays is due to a process going on throughout the rolume of the gas, and is not due to the disintegration of charged dust from the electrodes.

Cavendish Laboratory, December 28, 1896.

\section{Note on the preceding Paper, by J. J. 'Тномson.}

The connexion obtained by Mr. Rutherford between the coefficient of absorption and the saturation current through the gas admits of an interesting method of expression on the theory that the Röntgen rays so far resemble light as to be of the nature of an electromagnetic wave or impulse. We may regard such a wave or impulse as consisting of groups or a group of Faraday tubes travelling outwards through space. These tubes are of equal strength, the strength of each corresponding to the atomic charge carried by a univalent atom. If a molecule of the gas through which the rays are passing gets dissociated into ions by the electrio field produced by the tubes, one and only one of the tubes will get detached from the group and will be anchored by having its ends attached to the ions into which the molecule is dissociated. The dissociation of one molecule, or the production of one positive and one negative ion, will withdraw just one tube from those in the group forming the Röntgen rays. Now Mr. Rutherford's result, if we can extend it to all gases, shows that the production of each ion corresponds to a weakening of the Röntgen rays (by the same amount) whatever may be the gas from which the ion is formed. 'Ihe intensity of the rays is supposed to be measured by the conductivity they produce in a standard gas at standard temperature and pressure. Thus Mr. Rutherford's result may be expressed by saying that the weakening of the rays is proportional to the number of Faraday tubes stopped; and hence that the intensity of the rays is proportional to the number of Faraday tubes. 